\title{
What is the Optimal Time to Perform Cardiac Surgery in Patients with Stroke?
}

Timothy Hartman BS ${ }^{1}$, Mackenzie Madison MS ${ }^{1}$, Niha Namburi MHA ${ }^{1}$, Luke Haag ${ }^{1}$, and Lawrence Lee MD ${ }^{1}$

${ }^{1}$ Division of Cardiothoracic Surgery, Indiana University School of Medicine

Background: Preoperative stroke in patients undergoing cardiac surgery is a risk factor associated with increased postoperative complications, including stroke. The optimal timing when cardiac surgery can be safely performed after stroke is unknown. The purpose of this study was to investigate how cardiac surgery timing after preoperative stroke impacts postoperative outcomes.

Methods: All patients with history of stroke who subsequently underwent cardiac surgery at IU Health Methodist Hospital between 2011-2017 were retrospectively reviewed. Patient variables were extracted from both the institutional Society of Thoracic Surgeons (STS) database and a statewide Indiana patient database. Medical records of each patient were also reviewed. Patients were grouped into cohorts by the timing of the preoperative stroke relative to cardiac surgery: Recent (within two weeks before surgery), Intermediate (greater than two weeks but less than six weeks before), and Remote (greater than six weeks before). Postoperative outcomes were reviewed and compared between groups.

Results: 165 patients were included in the study, with 48 in the Recent group, 27 in Intermediate, and 90 in Remote. 30-day mortality and postoperative stroke rates, respectively, for the groups without endocarditis $(n=110)$ were: Recent $-0.0 \%$ and $28.6 \%$, Intermediate $-0.0 \%$ and $8.3 \%$, and Remote $-3.9 \%$ and $5.2 \%$. The difference in postoperative stroke rate between Recent and Remote was significant $(p=0.005)$ with patients in the Recent group estimated to be 7.3 times more likely to develop postoperative stroke in comparison to the Remote group, while the difference between Recent and Intermediate was not. Preoperative stroke due to endocarditis $(n=55)$ was not associated with a significant increase in postoperative stroke, regardless of timing of surgery.

Conclusion: Delaying cardiac surgery for six weeks after stroke occurrence may lead to significantly reduced likelihood of postoperative stroke. The exception would be patients with preoperative stroke due to septic emboli from endocarditis, as timing of cardiac surgery does not seem to affect postoperative mortality or stroke rates in these patients. 